\title{
The Inverse OARSI-OMERACT Criteria Is a Valid Indicator of the Clinical Worsening of Knee Osteoarthritis: Data From the Osteoarthritis Initiative
}

\author{
Jeffrey B. Driban ${ }^{1}$ (D), Matthew S. Harkey² (D), Lori Lyn Price ${ }^{3}$, Grace H. Lo ${ }^{4}$, and Timothy E. McAlindon ${ }^{1}$
}

\begin{abstract}
Objective. We assessed if the inverse Osteoarthritis Research Society International (OARSI) and Outcome Measures in Rheumatology (OMERACT) criteria relate to concurrent radiographic knee osteoarthritis (KOA) progression and decline in walking speed, as well as future knee replacement.

Methods. We conducted knee-based analyses of data from the Osteoarthritis Initiative. All knees had symptomatic OA: at least doubtful radiographic KOA (Kellgren-Lawrence grade $\geq 1$ ) and knee pain $\geq 10 / 100$ (Western Ontario and McMaster Universities Osteoarthritis Index pain) at the 12-month visit. The inverse of the OARSI-OMERACT responder criteria depended on knee pain and function, and global assessment of knee impact. We used generalized linear mixed models to assess the relationship of the inverse OARSI-OMERACT criteria over 2 years (i.e., 12-month and 36-month visits) with worsening radiographic severity (any increase in Kellgren-Lawrence grade from 12 months to 36 months) and decline in self-selected 20 -m walking speed of $\geq 0.1 \mathrm{~m} / \mathrm{s}$ (from 12 months to 36 months). We used a Cox model to assess time to knee replacement during the 6 years after the 36 -month visit as an outcome.

Results. Among the 1746 analyzed, 19\% met the inverse OARSI-OMERACT criteria. Meeting the inverse OARSI-OMERACT criteria was associated with almost double the odds of experiencing concurrent worsening in radiographic KOA severity (OR 1.89, 95\% CI 1.32-2.70) or decline in walking speed (OR 1.82, 95\% CI 1.37-2.40). A knee meeting the inverse OARSI-OMERACT criteria was more likely to receive a knee replacement after the 36-month visit (23\%) compared with a nonresponder (10\%; HR 2.54, 95\% CI 1.89-3.41).

Conclusion. The inverse OARSI-OMERACT criteria for worsening among people with KOA had good construct validity in relation to clinically relevant outcomes.
\end{abstract}

Key Indexing Terms: knee, osteoarthritis, pain, patient-reported outcome measures

The mandate to focus on patient-reported outcomes (PRO) and individual-specific outcomes requires a strategy to define clinically meaningful improvement and worsening of osteoarthritis (OA). While there exist validated methods to indicate clinically meaningful improvement, such as the Osteoarthritis Research Society International (OARSI) and Outcome Measures in Rheumatology (OMERACT) responder criteria set ${ }^{1}$, no complementary strategy to indicate worsening is
These analyses were financially supported by a public-private partnership comprised 5 contracts (NO1-AR-2-2258; NO1-AR-2-2259; NO1-AR-2-2260; N01-AR-2-2261; N01-AR-2-2262) funded by the National Institutes of Health (NIH), a branch of the Department of Health and Human Services, and conducted by the Osteoarthritis Initiative (OAI) Study Investigators. Private funding partners include Merck Research Laboratories, Novartis Pharmaceuticals Corporation, GlaxoSmithKline, and Pfizer Inc. Private sector funding for the OAI is managed by the Foundation for the NIH. This manuscript was prepared using an OAI public use dataset and does not necessarily reflect the opinions or views of the OAI investigators, the NIH, or the private funding partners. The project described was supported by the National Center for Advancing Translational Sciences, NIH, Award Number UL1TR002544. The content is solely the responsibility of the authors and does not necessarily represent the official views of the NIH. This work was also supported in part by the Houston Veterans Affairs Health Services Research and Development Center of Excellence (HFP90-020). The views expressed in this article are those of the authors and do not necessarily represent the views of the Department of Veterans Affairs. MSH was financially supported by a grant from the NIH (grant no. 5 TL1 TR 1454-3).

IJ.B. Driban, PhD, ATC, CSCS, Associate Professor, T.E. McAlindon,
MD, Chief of Rheumatology, Allergy, and Immunology, Division of Rheumatology, Allergy, and Immunology, Tufts Medical Center, Boston; ${ }^{2}$ M.S. Harkey, PhD, Postdoctoral Research Fellow, Division of Rheumatology, Allergy, and Immunology, Tufts Medical Center, Boston, and Department of Population and Quantitative Health Sciences, University of Massachusetts Medical School, Worcester; ${ }^{3}$ L.L. Price, MAS, Statistician, The Institute for Clinical Research and Health Policy Studies, Tufts Medical Center, and Tufts Clinical and Translational Science Institute, Tufts University, Boston, Massachusetts; ${ }^{4}$ G.H. Lo, MD, Assistant Professor, Medical Care Line and Research Care Line, Houston Health Services Research and Development (HSR\&D) Center of Excellence Michael E. DeBakey VAMC, and Section of Immunology, Allergy, and Rheumatology Baylor College of Medicine, Houston, Texas, USA.

The authors have no other conflicts of interest with regard to this work. Address correspondence to Dr. J.B. Driban, Division of Rheumatology, Tufts Medical Center, 800 Washington Street, Box 406, Boston, MA 02111, USA. Email:jeffrey.driban@tufts.edu.

Full Release Article. For details see Reprints and Permissions at jrheum.org. Accepted for publication May 26, 2020. 
widely accepted for research applications $s^{2,3,4,5,6,7}$. One possibility is to apply the inverse of the OARSI-OMERACT responder criteria to assess clinically meaningful worsening; however, this approach has not been validated in relation to clinically relevant worsening outcomes in people with knee OA (KOA).

In this brief report, we describe our efforts to assess if the inverse OARSI-OMERACT criteria for clinical worsening relates to concurrent radiographic KOA progression and slowed walking speed, as well as future knee replacement. Since the OARSI-OMERACT criteria is considered a gold standard for defining clinical improvement of OA, our goal is to demonstrate the construct validity of the inverse OARSI-OMERACT criteria as an indicator of clinical worsening OA.

\section{MATERIALS AND METHODS}

Study design. We conducted knee-based analyses of data from the Osteoarthritis Initiative (OAI). We assessed the inverse OARSI-OMERACT criteria, worsening radiographic KOA severity, and a decline in walking speed over 2 years (between the 12-month and 36-month visits). We also assessed if someone received a knee replacement during the 6 years after the 36-month visit.

The OAI is a prospective cohort study of 4796 adults with or at risk for symptomatic KOA from 4 clinical sites in the United States. Study staff recruited participants between February 2004 and May 2006. OAI data and protocols are freely available online ${ }^{8}$.

Participant selection. The selection process is summarized in Figure 1. We required all knees to have an inclusive definition of symptomatic OA: at least doubtful OA [Kellgren-Lawrence $(\mathrm{KL})$ grade $\geq 1$ ] and knee pain $\geq 10 / 100$ [Western Ontario and McMaster Universities Osteoarthritis Index (WOMAC) pain subscale] at the 12 -month visit. The KL grade defined radiographic severity and was based on blinded central readings of bilateral weight-bearing, fixed-flexion posteroanterior knee radiographs (intrarater agreement: weighted $\kappa 0.70-0.80)^{8,9}$. We excluded participants who had missing data that prevented us from determining if a knee met the inverse OARSI-OMERACT criteria, worsening radiographic KOA severity, or a decline in walking speed. Further, we excluded knees that received a joint replacement between the 12-month and 36-month visits.

Inverse OARSI-OMERACT criteria. The OARSI-OMERACT criteria relies on self-reported knee pain (knee-specific WOMAC pain), knee-related function (knee-specific WOMAC function), and patient global assessment of knee impact [Files: allclinical\#\# (versions 1.2.2 and 5.2.1) $]^{1,8,10,11}$. All scores were converted to $0-100$, with 100 being a poorer outcome. We calculated the 2-year absolute change (36-month visit data minus 12 -month visit data) and percent change. We selected a 2 -year observation period because 2 years is a common duration for a clinical trial for KOA. Figure 2 illustrates the decision rule for the inverse OARSI-OMERACT criteria. First, if a knee had worsening pain or function $\geq 50 \%$ and absolute change in pain or function $\geq 20$, then the knee was classified as worsening. If not, we classified a knee as worsening if it met at least 2 out of 3 criteria that relied on a worsening of $\geq 20 \%$ and an absolute change of $\geq 10 / 100$ for each outcome. If a knee failed to meet at least 2 of the criteria, then the knee had no worsening.

Worsening radiographic severity. We classified a knee with worsening radiographic severity if central readers reported any increase in KL grade between the 12-month and 36-month weight-bearing knee radiographs [File: kXR SQ_BU\#\#_SAS (versions 1.8 and 5.7) ] $]^{8}$. If a knee had no increase in the KL grade, then it was classified as having no radiographic severity worsening. Decline in walking speed. Decline in walking speed was based on change in

\section{Osteoarthritis Initiative $\mathrm{n}=9952$ knees}

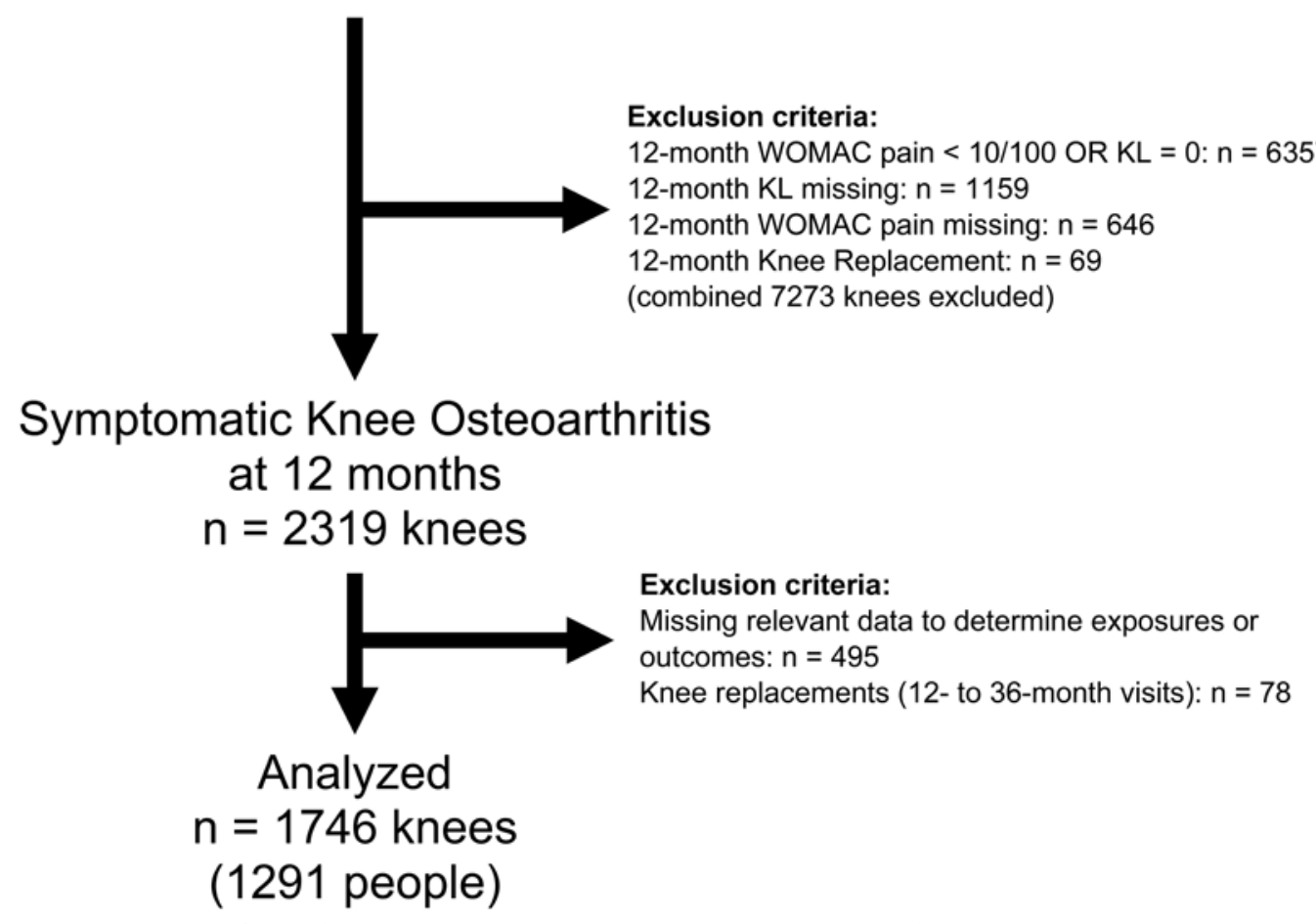

Figure 1. Flowchart for eligibility in analyses. KL: Kellgren-Lawrence arthritis grading scale; WOMAC: Western Ontario and McMaster Universities Osteoarthritis Index. 


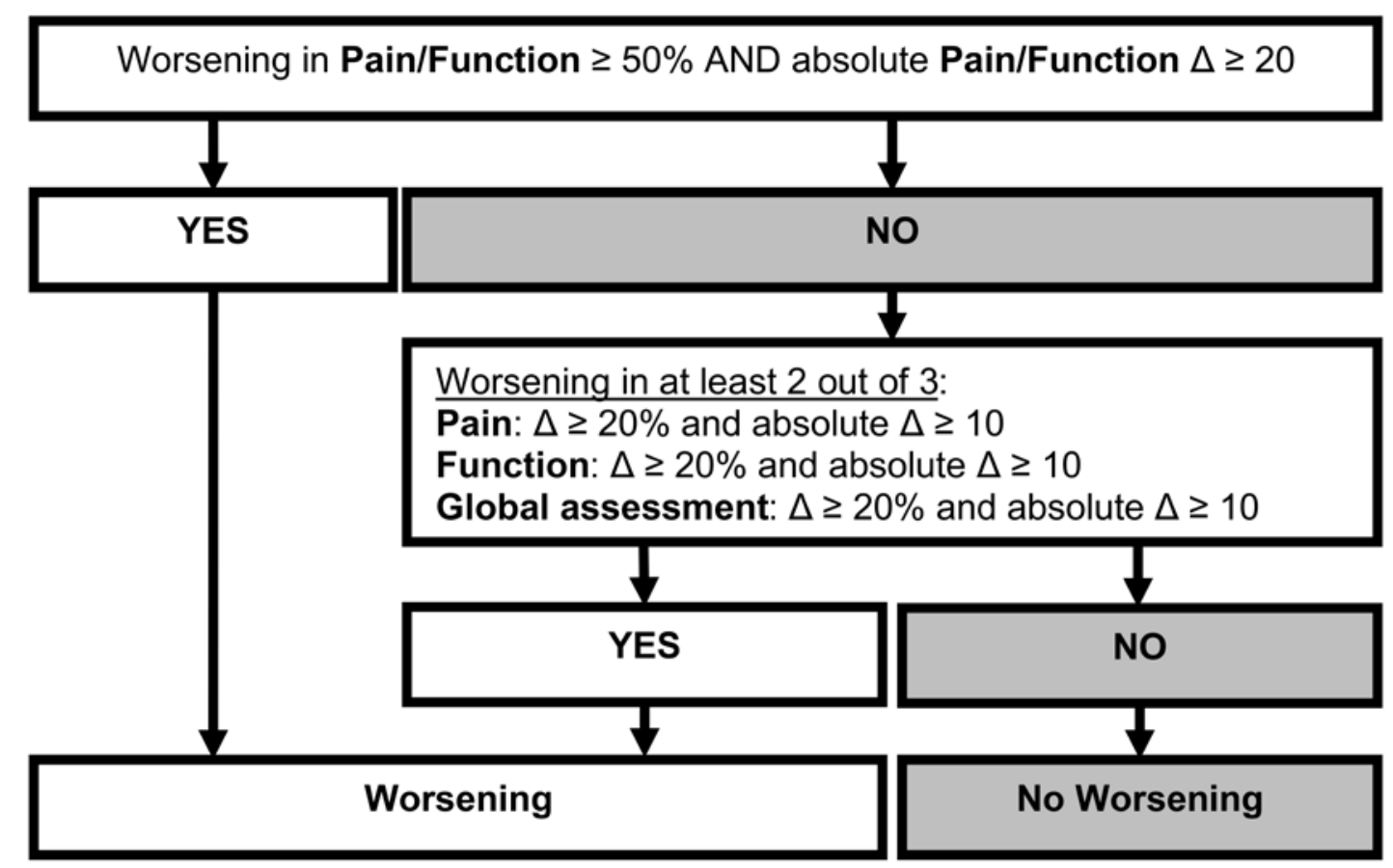

Figure 2. The inverse OARSI-OMERACT criteria for clinical worsening. $\Delta=$ Change. OARSI: Osteoarthritis Research Society International; OMERACT: Outcome Measures in Rheumatology.

the 20-meter walk speed between the 12-month and 36-month visits [Files: allclinical\#\# (versions 1.2.2 and 5.2.1) $]^{8}$. In brief, participants performed 2 trials of a 20 -meter walk at their usual, comfortable walking pace ${ }^{12,13}$. The time needed to walk 20 meters was converted to walking speed (i.e., $\mathrm{m} / \mathrm{s}$ ) and averaged across the 2 trials. We defined a decline in walking speed as someone who walked at least $0.1 \mathrm{~m} / \mathrm{s}$ slower at 36 months compared to 12 months ${ }^{12,14}$.

Knee replacement. We classified a knee with a future knee replacement if receipt of a knee replacement (partial or total) was reported or observed on radiographs in the 6 years after the time frame used to define the inverse OARSI-OMERACT criteria (12-month to 36 -month visit, $>98 \%$ adjudicated, 3 unadjudicated cases). Hence, a knee was classified with a knee replacement if it met 1 of 3 criteria for a knee replacement: (1) the knee replacement was centrally adjudicated ( 2 adjudicators reviewed medical records and a physician adjudicator was included if there was a disagreement between the first 2); (2) the knee replacement was observed on radiograph; or (3) the knee replacement was self-reported (even if the self-reported replacement had not gone through the adjudication process) [Files: outcomes99 (version 10)] $]^{8}$.

Statistical analysis. We evaluated the association of the inverse OARSI-OMERACT criteria with concurrent worsening radiographic severity and a decline in walking speed by performing 2 knee-based analyses using a logistic regression with repeated measures (generalized linear mixed model) to adjust for correlations between knees within person. The inverse OARSI-OMERACT criteria was the outcome for these analyses. To assess the association between the inverse OARSI-OMERACT criteria and time to knee replacement, we used a Cox model with a robust sandwich covariance matrix estimate to account for correlation between knees ${ }^{15}$. Our models were unadjusted because we were interested in the overall association between the inverse OARSI-OMERACT criteria and the other outcomes as a means of construct validity. We performed a sensitivity analysis among people with $\mathrm{KL} \geq 2$. All analyses were performed with SAS Enterprise 7.15 with a $P$ value $\leq 0.05$ to define statistical significance.

Ethical standards. The OAI was approved and meets all criteria for ethical standards regarding human studies defined in the 1964 Declaration of
Helsinki and all amendments made after. Institutional review boards at each clinical site and the coordinating center (University of California, San Francisco, USA) approved the OAI study (approval number 10-00532). All participants provided informed consent prior to participation.

\section{RESULTS}

We analyzed 1746 out of the 2319 (75\%) knees with symptomatic KOA at the 12 -month visit $(n=1291$ people; Figure 1$)$. On average, excluded knees had more severe knee symptoms than those included in the analyses (Table 1). Overall, 19\% of knees $(n=335)$ met the inverse OARSI-OMERACT criteria for worsening. Both responders and nonresponders were mostly female (62\% and 61\%, respectively) and White (70\% and $74 \%$, respectively). Table 1 offers additional insights in the group characteristics.

A knee meeting the inverse OARSI-OMERACT criteria for worsening was about twice as likely to experience concurrent worsening radiographic severity (OR 1.89, 95\% CI 1.32-2.70) or a decline in walking speed (OR 1.82, 95\% CI 1.37-2.40; see Table 1 for the frequency of each outcome by group).

A knee meeting the inverse OARSI-OMERACT criteria for worsening was also more likely to receive a knee replacement after the 36-month visit compared to a nonresponder (HR 2.54, 95\% CI 1.89-3.41; Table 1).

The results were similar when only including knees with $\mathrm{KL}$ $\geq 2(\mathrm{n}=1405)$.

\section{DISCUSSION}

The inverse OARSI-OMERACT criteria among people with KOA had good construct validity in relation to worsening of clinically relevant outcomes. Further, we found that $19 \%$ of knees met the criteria, which is comparable to prior reports 
Excluded Knees with Symptomatic

OA (With Missing Data), $\mathrm{n}=573$
OARSI-OMERACT Criteria

No Worsening, $\mathrm{n}=1411$
OARSI-OMERACT Criteria

Worsening, $\mathrm{n}=335$

\begin{tabular}{|c|c|c|c|}
\hline \multicolumn{4}{|l|}{ Baseline } \\
\hline Female, n (\%) & $347(61)$ & $859(61)$ & $209(62)$ \\
\hline Age, yrs & $64.0(9.3)$ & $62.3(9.0)$ & $63.4(9.0)$ \\
\hline WOMAC pain, $0-100$ & $31.6(18.1)$ & $25.7(15.8)$ & $22.8(12.7)$ \\
\hline WOMAC function, $0-100$ & $29.3(20.0), \mathrm{n}$ miss $=14$ & $22.8(17.0)$ & $22.2(15.1)$ \\
\hline Global impact, $0-100$ & $34.6(24.2)$ & $27.4(22.2)$ & $30.5(21.2)$ \\
\hline 2 & $217(38 \%)$ & $603(43 \%)$ & $116(35 \%)$ \\
\hline 3 & $152(27 \%)$ & $389(28 \%)$ & $123(37 \%)$ \\
\hline 4 & $113(20 \%)$ & $131(9 \%)$ & $43(13 \%)$ \\
\hline \multicolumn{4}{|l|}{ Concurrent worsening, $12-36$ months } \\
\hline Worsening radiographic severity & ND & $121(9 \%)$ & $52(16 \%)$ \\
\hline Decline in walking speed, $\leq 0.1 \mathrm{~m} / \mathrm{s}$ & ND & $289(20 \%)$ & $107(32 \%)$ \\
\hline
\end{tabular}

Means (SD) reported unless noted otherwise. ${ }^{a}$ Radiographic severity worsening defined as any increase in KL grade. KL: Kellgren-Lawrence; $\mathrm{n}$ miss $=$ number of knees with missing data for a variable; ND = no data reported with > 340 knees missing data. OA: osteoarthritis; OARSI: Osteoarthritis Research Society International; OMERACT: Outcome Measures in Rheumatology; WOMAC: Western Ontario and McMaster Universities Osteoarthritis Index.

of self-reported worsening $(9-29 \%)^{2,45,6}$. Hence, the inverse OARSI-OMERACT responder criteria may be appropriate for use in epidemiological studies.

While other investigators have analyzed the minimal clinically meaningful difference in a single PRO for worsening, this approach offers a crude strategy that fails to account for the multiple dimensions of clinical symptoms ${ }^{2,3,5}$. Recently, investigators found that this inverse OARSI-OMERACT criteria performed well for specificity (0.87) but had a low sensitivity (0.22) to detect people reporting at least slight worsening after 3 months of multimodal treatment ${ }^{4}$. Hence, the criteria are a conservative marker of clinically meaningful worsening that successfully excludes people without meaningful worsening but misses some people who perceive worsening symptoms. In the current study, we also demonstrated that these criteria had good construct validity.

Unfortunately, we were unable to test the sensitivity and specificity of the criteria in this cohort because we lacked questions about transitions in symptom states. Further, we relied on PRO measures from 2005 (first 12-month visits) to 2009 (last 36-month visits) and knee replacements from 2007 to 2014, which may affect the generalizability of these findings to the current population. While the effect over time is likely minimal, future studies should monitor the validity of the inverse OARSI-OMERACT criteria as clinical practice changes. Finally, we focused on just 1 set of criteria to define clinical worsening because we believe it is valuable to adopt a definition for worsening that is comparable to one used to define improvement.

In conclusion, the inverse OARSI-OMERACT criteria to define worsening clinical outcomes among people with KOA had good construct validity and may be useful in longitudinal studies.

\section{REFERENCES}

1. Pham T, van der Heijde D, Altman RD, Anderson JJ, Bellamy N, Hochberg M, et al. OMERACT-OARSI initiative: Osteoarthritis Research Society International set of responder criteria for osteoarthritis clinical trials revisited. Osteoarthritis Cartilage 2004;12:389-99.

2. Angst F, Benz T, Lehmann S, Aeschlimann A, Angst J. Multidimensional minimal clinically important differences in knee osteoarthritis after comprehensive rehabilitation: a prospective evaluation from the Bad Zurzach Osteoarthritis Study. RMD Open 2018;4:e000685.

3. Mills KAG, Naylor JM, Eyles JP, Roos EM, Hunter DJ. Examining the minimal important difference of patient-reported outcome measures for individuals with knee osteoarthritis: a model using the knee injury and osteoarthritis outcome score. J Rheumatol 2016;43:395-404.

4. Mahler EA, den Broeder AA, Woodworth TG, Busch V, van den Hoogen FH, Bijlsma JW, et al. How should worsening in osteoarthritis be defined? Development and initial validation of preliminary criteria for clinical worsening in knee and hip osteoarthritis. Scand J Rheumatol 2017;46:396-406.

5. Angst F, Aeschlimann A, Michel BA, Stucki G. Minimal clinically important rehabilitation effects in patients with osteoarthritis of the lower extremities. J Rheumatol 2002;29:131-8.

6. Eyles JP, Mills K, Lucas BR, Williams MJ, Makovey J, Teoh L, et al. Can we predict those with osteoarthritis who will worsen following a chronic disease management program? Arthritis Care Res 2016;68:1268-77.

7. Alschuler KN, Molton IR, Jensen MP, Riddle DL. Prognostic value of coping strategies in a community-based sample of persons with chronic symptomatic knee osteoarthritis. Pain 2013;154:2775-81.

8. The Osteoarthritis Initiative. [Internet. Accessed November 11, 2020.] Available from: https://nda.nih.gov/oai

9. Felson DT, Niu J, Guermazi A, Sack B, Aliabadi P. Defining radiographic incidence and progression of knee osteoarthritis: suggested modifications of the Kellgren and Lawrence scale. Ann Rheum Dis 2011;70:1884-6. 
10. Dougados M, Leclaire P, van der Heijde D, Bloch DA, Bellamy N, Altman RD. Response criteria for clinical trials on osteoarthritis of the knee and hip: a report of the osteoarthritis research society international standing committee for clinical trials response criteria initiative. Osteoarthritis Cartilage 2000;8:395-403.

11. Bingham CO 3rd, Bird SR, Smugar SS, Xu X, Tershakovec AM. Responder analysis and correlation of outcome measures: pooled results from two identical studies comparing etoricoxib, celecoxib, and placebo in osteoarthritis. Osteoarthritis Cartilage 2008;16:1289-93.

12. Herzog MM, Driban JB, Cattano NM, Cameron KL, Tourville TW, Marshall SW, et al. Risk of knee osteoarthritis over 24 months in individuals who decrease walking speed during a 12-month period: data from the Osteoarthritis Initiative. J Rheumatol 2017; 44:1265-70.
13. White DK, Niu J, Zhang Y. Is symptomatic knee osteoarthritis a risk factor for a trajectory of fast decline in gait speed? Results from a longitudinal cohort study. Arthritis Care Res 2013;65:187-94.

14. Motyl JM, Driban JB, McAdams E, Price LL, McAlindon TE. Test-retest reliability and sensitivity of the 20-meter walk test among patients with knee osteoarthritis. BMC Musculoskelet Disord 2013;14:166.

15. Lee EW, Wei LJ, Amato DA. Cox-type regression analysis for large numbers of small groups of correlated failure time observations. In: Klein JP, Goel PK, editors. Survival analysis: state of the art. Dordrecht, Netherlands: Kluwer Academic Publishers; 1992:237-47. 